\title{
PHOTOMETRIC PROPERTIES OF AGNS FROM THE ABSOLUTE SPECTROPHOTOMETRY OF DE BRUYN AND SARGENT
}

\author{
M. Kalinkov ${ }^{1}$, I. Kuneva ${ }^{1}$, Z. Tsvetanov ${ }^{1,2}$ and L. Filipov ${ }^{3}$ \\ 1 Dept. Astronomy, Bulgarian Acad. Sci., 72 Lenin Blvd., \\ 21784 Sofia, Bulgaria \\ ESO, Karl-Schwarzschild-Str. 2, D-8046 Garching bei Muenchen, \\ 3 F.R.G. \\ 3 Space Res. Inst., Bulgarian Acad. Sci., 8 Moskovska Str., \\ 1000 Sofia, Bulgaria
}

The absolute spectrophotometry of 66 AGNs (de Bruyn and Sargent, 1978) is used to compute synthetic magnitudes and colors, and K-corrections in the UBV system. In our preliminary contribution (Kalinkov et al., 1988) only some results of $(\mathrm{U}-\mathrm{B})_{s}$ and $(\mathrm{B}-\mathrm{V})_{s}$ colors were given. Here we use the whole observational data given by de Bruyn and Sargent. Synthetic spectra for 41 Sy1, 7 Sy1.5 and 15 Sy2 galaxies are constructed in relative units, assuming $f(\lambda 5470)=1000$. Colors and $\mathrm{K}$-corrections are obtained from the synthetic spectra. The variations with the redshift are presented in the polynomial approximation form $\mathrm{X}=\mathrm{a}_{i} \mathrm{z}^{i}, \mathrm{i}=0,1, .$. , where $\mathrm{X}=(\mathrm{U}-\mathrm{B})_{s s},(\mathrm{~B}-\mathrm{V})_{s s}, \mathrm{~K}_{B}, \mathrm{~K}_{V}$ (Table 1). The largest deviation in mag. from the computed curves is denoted by $Q$. The relations in Table 1 are very good approximations in the corresponding redshift ranges.

Table 1. Polynomial approximation $\mathrm{X}=\mathrm{a}_{i} \mathrm{z}^{i}$ for colors and $\mathrm{K}$-corrections

\begin{tabular}{|c|c|c|c|c|c|c|c|c|}
\hline $\mathrm{X}$ & Sy & $a_{0}$ & $a_{1}$ & $a_{2}$ & $a_{3}$ & $a_{4}$ & Q, mag. & Range \\
\hline$(\mathrm{U}-\mathrm{B})_{s}$ & 1 & -0.671 & -1.220 & & & & 0.01 & $0 \leq \mathrm{z} \leq 0.12$ \\
\hline$(\mathrm{B}-\mathrm{V})_{s}$ & 1 & 0.391 & 1.037 & -6.09 & & & 0.01 & $0 \leq \mathrm{z} \leq 0.26$ \\
\hline$(\mathrm{B}-\mathrm{V})_{s}$ & 1.5 & 0.566 & 2.900 & -12.49 & & & 0.005 & $0 \leq \mathrm{z} \leq 0.18$ \\
\hline$(\mathrm{B}-\mathrm{V})_{s}$ & 2 & 0.708 & 4.031 & -12.82 & & & 0.005 & $0 \leq \mathrm{z} \leq 0.18$ \\
\hline $\mathrm{K}_{B}$ & 1 & 0.005 & 0.300 & -2.41 & & & 0.01 & $0<z \leq 0.32$ \\
\hline $\mathrm{K}_{B}$ & 1.5 & -0.005 & 1.975 & -4.12 & & & 0.01 & $0<\mathrm{z} \leq 0.18$ \\
\hline $\mathrm{K}_{B}$ & 2 & -0.003 & 3.048 & -0.89 & & & 0.01 & $0<\mathrm{z} \leq 0.18$ \\
\hline$K_{V}$ & 1 & 0.004 & -1.496 & 12.14 & -27.71 & 18.74 & 0.015 & $0<\mathrm{z} \leq 0.66$ \\
\hline $\mathrm{K}_{V}$ & 1.5 & -0.022 & -0.440 & 8.05 & -10.46 & & 0.03 & $0.03<\mathrm{z} \leq 0.52$ \\
\hline $\mathrm{K}_{V}$ & 2 & -0.044 & 0.112 & 7.85 & 8.32 & & 0.025 & $0.04<\mathrm{z} \leq 0.54$ \\
\hline
\end{tabular}

The colors $(\mathrm{B}-\mathrm{V})_{s s}, \mathrm{~K}_{B}$ and $\mathrm{K}_{V}$, calculated from the synthetic spectra are given in Figs 1-3 with the st.dev. of the mean, $\sigma / \sqrt{n}$.

de Bruyn, A. G. and Sargent, W. L. W. 1978, A.J., 83, 1257.

Kalinkov, M., Kuneva, I., Tsvetanov, Z. and Filipov, L. 1988, Adv. Space Res., 8, 75 . 


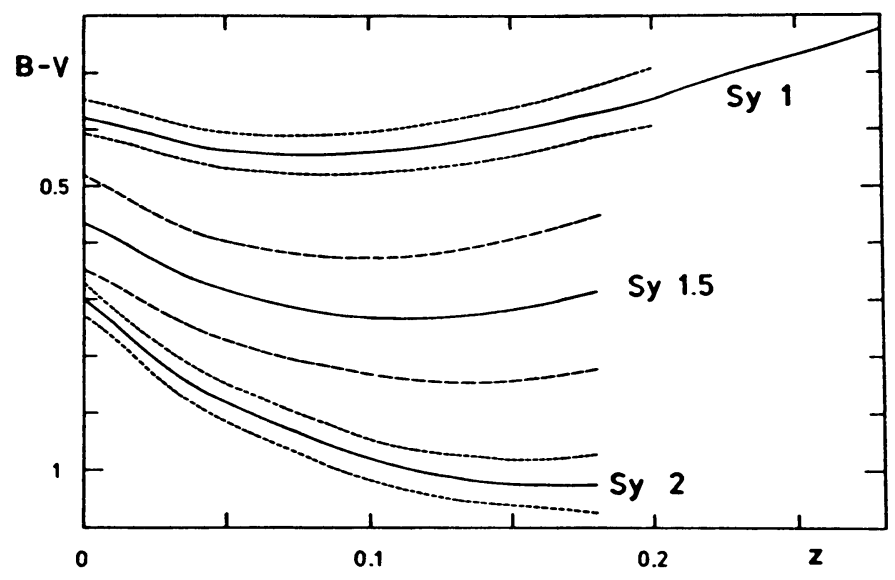

Fig. 1 .

Colors $(B-V)$ ss

from the

Fig. 2. $\mathrm{K}_{\mathrm{B}}$-correction from

the synthetic

spectra.
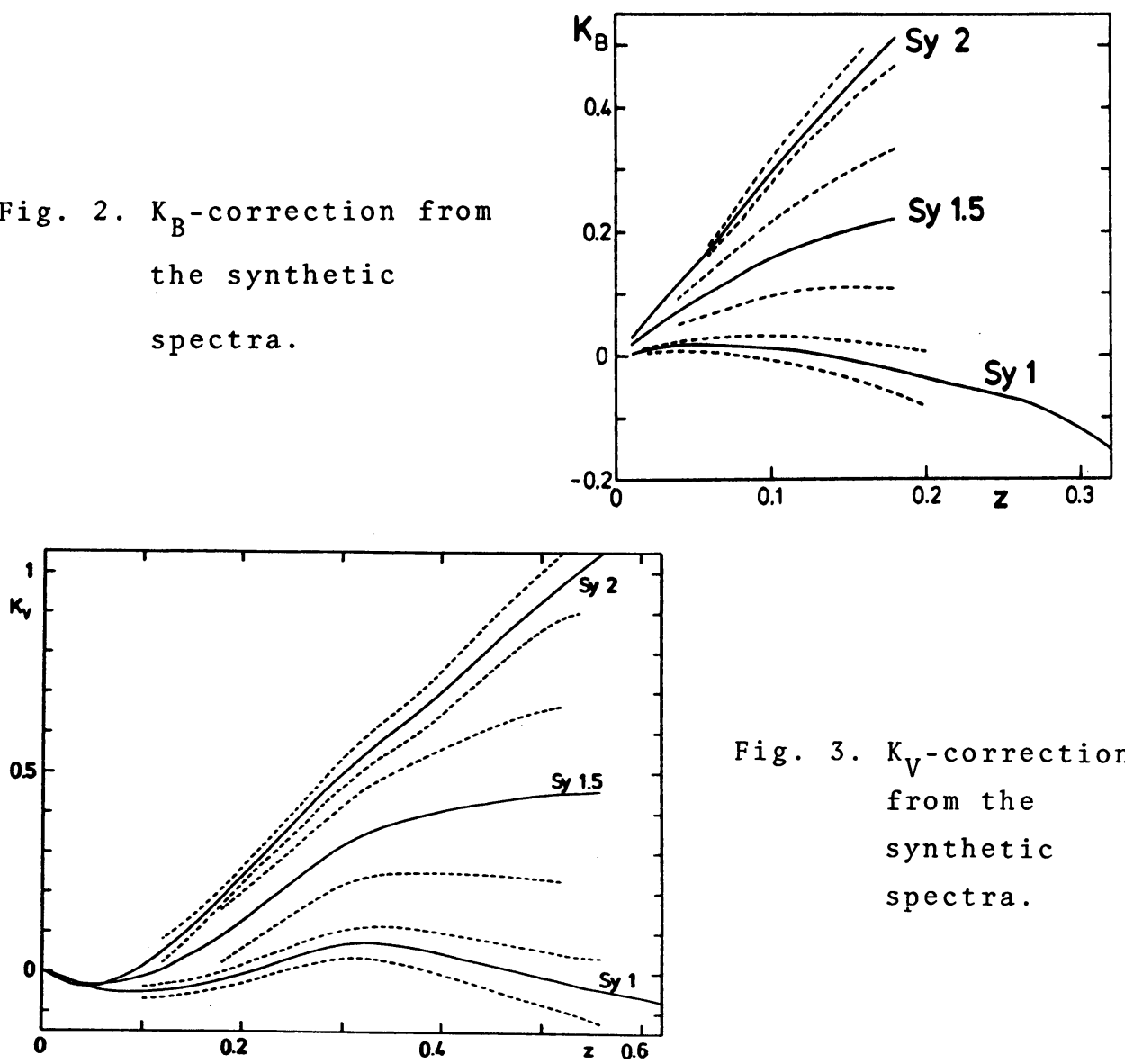

Fig. 3. $\mathrm{K}_{\mathrm{V}}$-correction from the synthetic spectra. 